\title{
Interfacial Interactions and Nanostructure Changes in DPPG/HD Monolayer at the Air/Water Interface
}

\author{
Huaze Zhu, ${ }^{1,2}$ Runguang Sun, ${ }^{1}$ Tao Zhang, ${ }^{2}$ Changchun Hao, ${ }^{1}$ Pengli Zhang, ${ }^{1,2}$ \\ Juan Wang, ${ }^{1}$ and Shaorong $\mathrm{Li}^{2}$ \\ ${ }^{1}$ College of Physics and Information Technology, Shaanxi Normal University, Chang-an Street No. 199, Xian 710062, China \\ ${ }^{2}$ College of Science, Xian University of Science and Technology, Yanta Road No. 58, Xian 710054, China
}

Correspondence should be addressed to Runguang Sun; sunrunguang@snnu.edu.cn and Tao Zhang; tzhang-psu@yahoo.com

Received 8 September 2015; Accepted 7 October 2015

Academic Editor: Jun Chen

Copyright (C) 2015 Huaze Zhu et al. This is an open access article distributed under the Creative Commons Attribution License, which permits unrestricted use, distribution, and reproduction in any medium, provided the original work is properly cited.

Lung surfactant (LS) plays a crucial role in regulating surface tension during normal respiration cycles by decreasing the work associated with lung expansion and therefore decreases the metabolic energy consumed. Monolayer surfactant films composed of a mixture of phospholipids and spreading additives are of optional utility for applications in lung surfactant-based therapies. A simple, minimal model of such a lung surfactant system, composed of 1,2-dipalmitoyl-sn-glycero-3-[phosphor-rac-(1-gylcerol)] (DPPG) and hexadecanol (HD), was prepared, and the surface pressure-area $(\pi-A)$ isotherms and nanostructure characteristics of the binary mixture were investigated at the air/water interface using a combination of Langmuir-Blodgett (LB) and atomic force microscopy (AFM) techniques. Based on the regular solution theory, the miscibility and stability of the two components in the monolayer were analyzed in terms of compression modulus $\left(C_{s}^{-1}\right)$, excess Gibbs free energy $\left(\Delta G_{\text {exc }}^{\pi}\right)$, activity coefficients $(\gamma)$, and interaction parameter $(\xi)$. The results of this paper provide valuable insight into basic thermodynamics and nanostructure of mixed DPPG/HD monolayers; it is helpful to understand the thermodynamic behavior of HD as spreading additive in LS monolayer with a view toward characterizing potential improvements to LS performance brought about by addition of HD to lung phospholipids.

\section{Introduction}

Monolayer of mixed amphiphiles has been extensively investigated as membrane models to interpret the physical and chemical behavior of monolayer and gain information about the structural changes of the monolayer induced by molecular lateral packing [1]. In particular, it is an effective tool to study intermolecular interactions between membrane molecules and HD. Researchers have applied various methods to acquire more information on lipids/HD monolayer properties, such as Langmuir-Blodgett technology, synchrotron X-ray diffraction, Fourier transforming infrared spectrum, Brewster Angle microscopy, and atomic force microscopy [2-6]. Among them, the LB technology has been widely used as the most effective way to prepare nanometer order monolayers and acquire the information on intermolecular interactions [7]. It also can achieve the molecular assembly by means of changing the length of hydrophobic chain or insetting ion to operate the structure, film thickness, orientation, and sequences of the monolayer precisely. On the other hand, the AFM has become an important technology which characterizes surface morphology of monolayer by utilizing the atomic interaction between the sample surface and the scanning probe [8].

Lung surfactant, a complex mixture of lipids and proteins found in the alveoli and affiliated bronchial interfaces, plays a crucial role in regulating surface tension during normal respiration cycles by decreasing the work associated with lung expansion during respiration and therefore decreases the metabolic energy consumed. Production insufficient or faulty LS in premature infants is a major cause of mortality and morbidity and consequently its biophysical action has been and still is the subject of numerous studies [9]. While useful, many LS preparations are made from animal extracts which 
can undergo large bath-to-batch variation as well as concerns over potential zoonotic diseases; hence there are advantages in developing purely synthetic LS exogenous surfactant preparations for medical applications. Hexadecanol, acting as a spreading agent, has been used in exogenous surfactant preparations for partially overcoming the bad spreading of lung surfactant lipids and it makes a positive contribution to surfactant performance [10]. In previous reports $[9,11]$, the mixed monolayer behavior of dipalmitoyl phosphatidylcholine (DPPC), which is the predominant lipid component of natural lung surfactant, with HD at the air/water interface as a function of mole fraction of HD, has been analyzed, and the results illustrate that the binary monolayer appears to be miscible, and the HD improves the surface tension kinetics of DPPC. It has been also found that the molecular packing in mixed DPPC/HD monolayers may be favored by the packing efficiency or geometric accommodation at higher surface pressure [11]. Grazing incidence X-ray diffraction data shows that the HD preferentially interacts with DPPC when HD is added into Infasurf (one clinical lung surfactant preparation). HD intercalates between the DPPC chains and hence leads to greater stability of the solid phase and a tighter packing of the two-dimensional lattice compared with pure system [10]. While most investigations on the behavior of HD in LS model membranes were performed using DPPC, the lung surface monolayers are composed of phospholipids with different polar headgroups and hydrocarbon tails. Besides DPPC, the anionic phospholipid DPPG is another important LS surfactant, which has superior adsorption and spreading properties in comparison with that of DPPC [12], accounting for about $5 \%$ of the total surfactant phospholipid pool with most species [13]. As we know, DPPG and DPPC have the same hydrocarbon tails, but the DPPC has net neutral charge and larger headgroup, which are very different from those of the DPPG. Different conformations of the polar headgroups of phospholipids may lead to differences in the behavior of monolayer with the HD incorporation. But the binary system of DPPG/HD has been not given the adequate attention in monolayer researches. The aim of this work is to investigate the DPPG/HD binary Langmuir monolayers spreading on pure water from the view of thermodynamics and physical chemistry by using LB and AFM technique. In order to establish the mutual miscibility and the molecular interaction between DPPG and HD at the interface, the mean molecular area $(A)$, excessive molecular area $\left(A_{\text {exc }}\right)$, compression modulus, excessive Gibbs free energy, interaction parameter, and activity coefficients have been quantitatively evaluated based on the experimental $\pi$-A isotherms recorded by KSV Minitrough instrument. The investigation of interaction between DPPG and HD in molecular level gives significant information and experimental basis for elucidating and understanding the underlying molecular mechanism between HD and lung surfactant lipid DPPG. Although the mixed monolayer behavior might become complex, because nonnegligible desorption of the monolayers might occur [11, 14], desorption of DPPG or HD, if there is any, was ignored in the analysis.

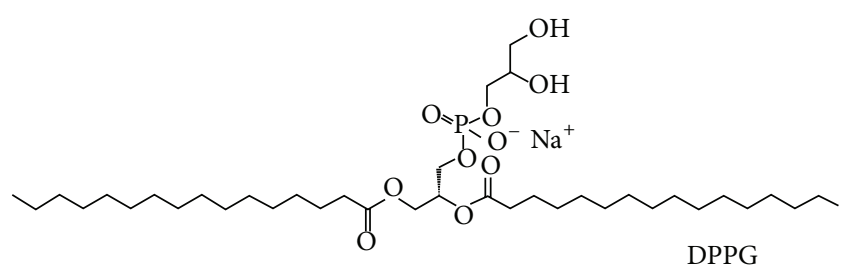

(a)

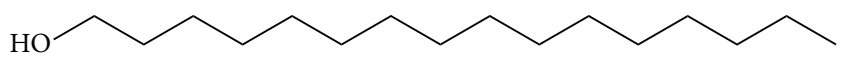

HD

(b)

FIgure 1: Chemical structures of (a) DPPG and (b) HD.

\section{Materials and Methods}

2.1. Materials. Synthetic sodium salt of 1,2-dipalmitoyl-snglycero-3-[phosphor-rac-(1-glycerol)] (DPPG, purity > 99\%) was obtained from Avanti Polar Lipids, Inc. (Alabaster, Al, USA). The HD (1-hexadecanol, purity > 99\%) was purchased from Sigma (St. Louis, USA). The molecular structures of the amphiphilic molecules are shown in Figure 1. The two components were dissolved in chloroform (HPLC grade) and diluted to $1 \mu \mathrm{mol} / \mathrm{mL}$, respectively, before they were spread at the air-water interface. A few drops of methanol (HPLC grade) were used to aid the dissolution of DPPG into chloroform. All preparations were stored at $4^{\circ} \mathrm{C}$. The subphase for all the experiments is ultrapure water (resistivity $>18 \mathrm{M} \Omega \mathrm{cm}$ ). The surface tension of the purified water was about $72 \mathrm{mN} / \mathrm{m}$ as measured by a Wilhelmy plate tensiometer at $25^{\circ} \mathrm{C}$. All glass wares used in the experiment in contact with the samples were rinsed with purified water.

2.2. Isotherm Data Collection. An automatic controlled KSV Minitrough (KSV Instruments Ltd., Finland) was used to obtain the $\pi$-A isotherms and LB monolayer at the airwater interface. The instrumentation is detailed elsewhere $[15,16]$ and summarized below. The apparatus consists of a Teflon trough fitted with two symmetrically mobile barriers and a Wilhelmy plate used as the surface balance probe. The measurements were performed with an accuracy of $\pm 0.004 \mathrm{mN} / \mathrm{m}$ in surface pressure and $\pm 1 \%$ in surface area, according to the instrument specification. Before each run, the Teflon trough was washed with ethanol and rinsed with purified water. The platinum plate was cleaned between each experiment by rinsing with purified water and heating to red heat. For all the experiments, the trough was filled with purified water as the subphase, and the temperature was maintained at $25 \pm 0.5^{\circ} \mathrm{C}$ by an external circulator. The given molecules (DPPG and HD) were mixed and six different stoichiometries $\left(X_{\mathrm{HD}}=0,0.2,0.4,0.6,0.8,1\right)$ were studied. The mixed DPPG/HD solution was spread dropwise and equably at the air/water interface with a Hamilton microliter syringe after the interface was cleaned. Fifteen minutes was allowed for solvent evaporation and monolayer equilibration before an experiment was started. Then the monolayer at the air-water interface was continuously compressed at a rate of 
$0.01 \mathrm{~nm}^{2}$ molecule ${ }^{-1} \mathrm{~min}^{-1}$ to obtain the $\pi-A$ isotherms. As a monolayer was compressed to be in a condensed phase, the isotherm generally exhibited a sharp break (which was mentioned as the collapse point of the monolayer under the given experimental condition) followed by an abnormal change of surface pressure upon further compression. To ensure the reproducibility, each group of data was repeated at least twice (the error for the area per molecule and surface pressure does not exceed $0.002 \mathrm{~nm}^{2} /$ molecule and $0.1 \mathrm{mN} / \mathrm{m}$, resp.).

2.3. Atomic Force Microscopy. The AFM methods have been described in detail in previous studies $[8,17]$, and the main steps can be summarized as follows: freshly cleaved mica was used as a supporting solid substrate for film deposition. The mixed monolayers were compressed up to the given surface pressure. The deposition was carried out after the monolayer stabilization $15 \mathrm{~min}$ with the transferring rate of $1 \mathrm{~mm} / \mathrm{min}$. AFM images were obtained using an SPM-9500-J3 atomic force microscopy (Shimadzu Instruments Co. Ltd., Japan). The contact mode images (256 or 512 points per line) were collected with scan rates of $0.5-1 \mathrm{~Hz}$, using a micro V-shaped cantilever (Olympus Optical Co. Ltd., Japan) with a spring constant of $0.06 \mathrm{~N} / \mathrm{m}$, a thickness of $400 \mathrm{~nm}$, and a length of $100 \mu \mathrm{m}$. The lateral and vertical resolutions were 0.2 and $0.01 \mathrm{~nm}$, respectively. The transferred samples were checked for possible tip-induced deformation by zooming out after a region had been scanned.

\section{Results and Discussion}

3.1. $\pi$-A Curves at Discrete Mole Fraction. Figure 2 shows $\pi$ $A$ curves of DPPG/HD binary mixed monolayers formed at the air/water interface with various mole fractions of $\mathrm{HD}$, respectively. The behaviors of the isotherms recorded for a one-component monolayer (DPPG and HD films, resp.) are in agreement with previous studies $[9,15,18]$. In Figure 2 , the $\pi-A$ curve of the pure system of DPPG demonstrates that $\pi$ starts to go up with compression at the limiting surface area $A_{0}(\mathrm{DPPG})=0.5330 \mathrm{~nm}^{2}$ per molecule and reach a break point where a phase transition, as a plateau on the isotherm can be observed, from liquid-expand phase (LE) to liquidcondensed phase (LC) takes place $(\pi=10.91 \mathrm{mN} / \mathrm{m}, A=$ $0.6887 \mathrm{~nm}^{2}$ per molecule). The similar results were obtained by other researchers $[18,19]$. Interestingly, the transition point almost disappeared in the $\pi-A$ curve of DPPG onecomponent monolayer on pure water subphase at lower temperature $[20,21]$. These characteristic values as well as the collapse pressure obtained at the breaking of the isotherm curve are similar to those reported earlier [18]. It is needed to note that the collapse pressures of pure DPPG is obviously higher than that of HD, and this may be due to the fact that the headgroup of DPPG molecule is bigger than that of HD molecules, and the lipids used in the experiments have two hydrophobic tail chains, while a HD molecule only has single tail chain.

As is seen, $\pi-A$ curves for mixed DPPG/HD monolayers (see Figure 2) appeared in the order between those of both single systems and their shape varies systematically with

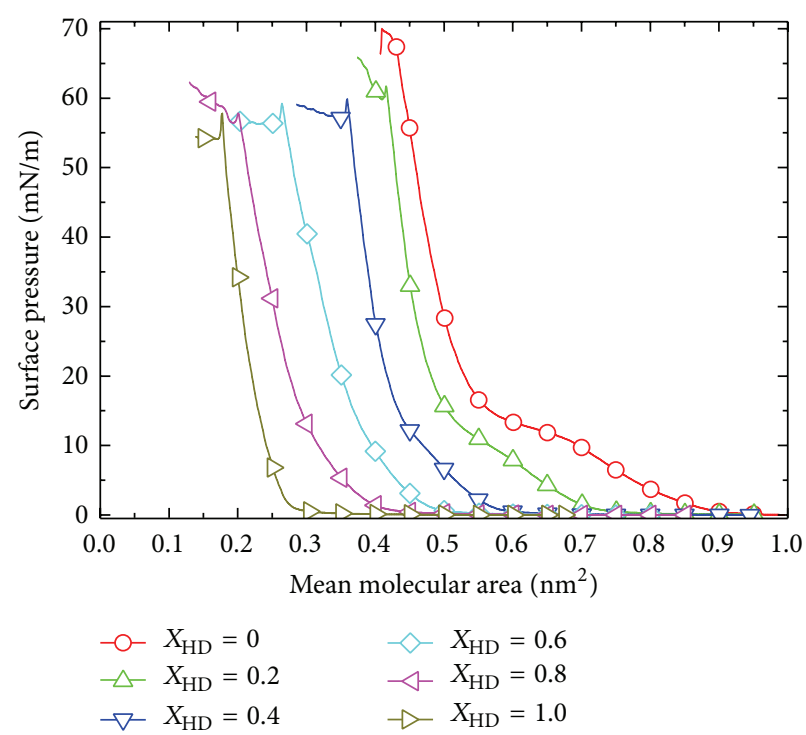

FIGURE 2: $\pi$ - $A$ isotherms of mixed DPPG/HD monolayers at discrete mole fraction of $X_{\mathrm{HD}}$ on the pure water at $25 \pm 0.5^{\circ} \mathrm{C}$.

the HD mole fraction, becoming similar to HD monolayer isotherm. The addition of HD into the phospholipid film makes the $\pi$-A curves steeper and provokes their shift toward smaller areas. This strong influence of HD on phospholipid monolayers is deemed the intercalating effect, that is, HD intercalates between the phospholipid double chains, and leads to a tighter packing of the two-dimensional lattice compared with single component system [10]. Figure 2 shows the break upon transition from LE to LC was observable up to $X_{\mathrm{HD}}=0.4$; such phenomena also occurred in the DPPC/HD binary system at $22^{\circ} \mathrm{C}$ [9]. According to the surface rule developed by Crisp from the ordinary phase rule in bulk solution [22], which is an effective way to establish miscibility of the binary system, if the components are miscible, the values of collapse pressure $\left(\pi_{c}\right)$ depend on the composition of its components and $\pi_{c}$ of the binary film lies between the collapse pressures of single components [23]. As Figure 2 has shown, $\pi_{c}$ for mixtures of DPPG/HD lies between the pure DPPG and HD, and the values of $\pi_{c}$ decrease with the increment of mole fraction of HD; it suggests that the DPPG and $\mathrm{HD}$ are miscible in the monolayer.

3.2. Miscibility of Binary Monolayers. It is well accepted that a completely immiscible monolayer and an ideal mixed film are absolutely opposite $[8,24,25]$. However, both obey (1). In a completely immiscible mixed monolayer, the intermolecular forces $F_{11} \gg F_{12} \ll F_{22}$ while in an ideal binary system, $F_{11}=F_{12}=F_{22}$, where $F_{i j}$ denote attractive forces between molecules of the two components $i$ and $j(i, j=1,2$, resp.) [26].

To determine the miscibility of the mixture and how the individual components of HD and DPPG are interacting with each other in the monolayer, one appropriate parameter is the evolution of mean molecular areas with the composition mole fraction at a concerned surface pressure. The variation 

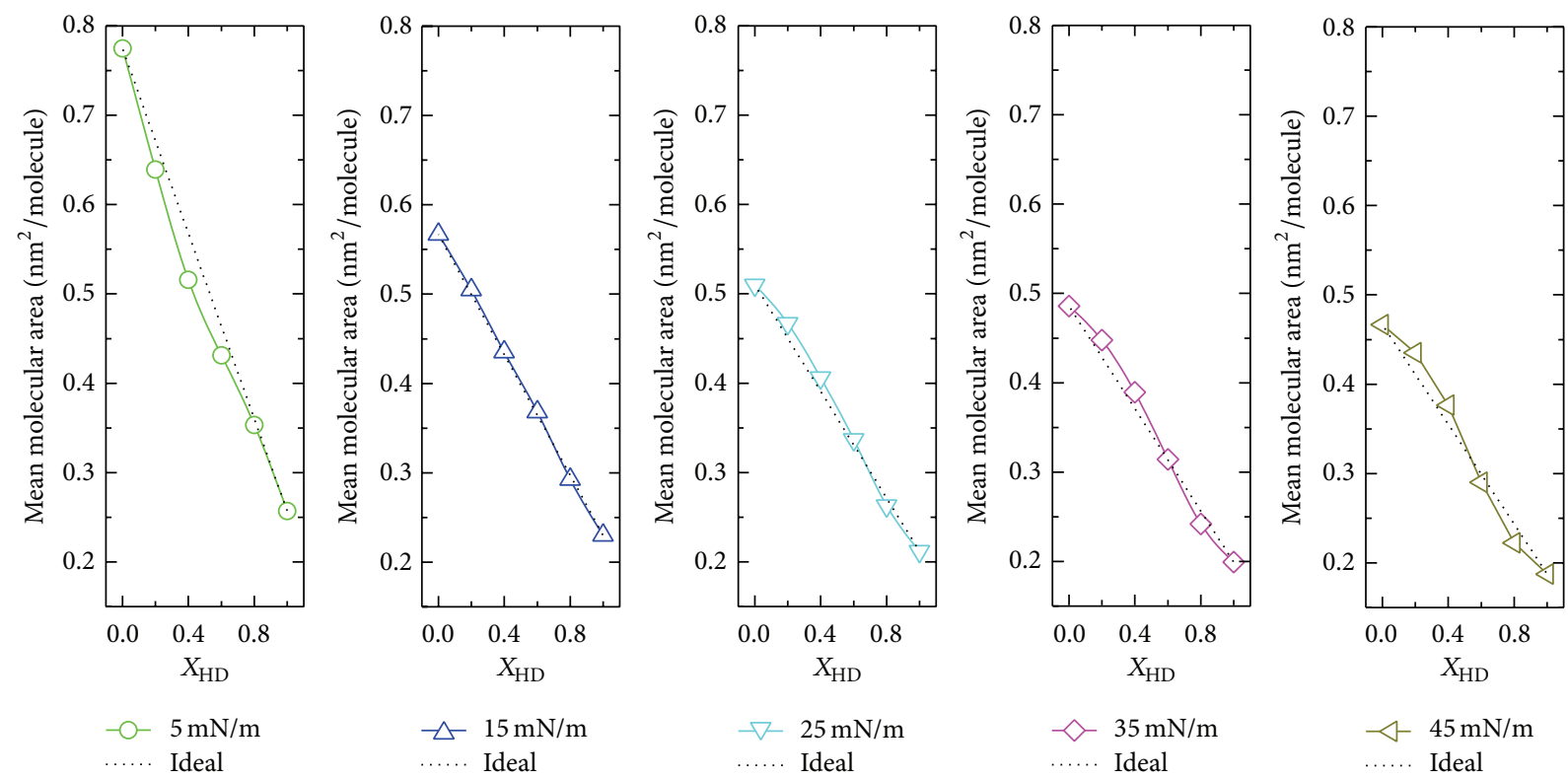

FIGURE 3: Mean molecule area as a function of composition of HD for mixed DPPG/HD monolayers on water subphase at discrete surface pressure (full line: real mean molecular area; dotted line: ideal mean molecular area).

of the experimental mean molecular area at $5,15,25,35$, and $45 \mathrm{mN} / \mathrm{m}$ for binary monolayers was plotted against the mole fraction of HD in Figure 3. According to the additivity rule $[23,27,28]$, the ideal value of the molecular area for the mixed DPPG/HD monolayers, $A_{\text {id }}$, can be calculated from the molar fraction of the two components, and the plot of $A_{\text {id }}$ versus $X_{1}$ will give a straight line:

$$
A_{\text {id }}=A_{1} X_{1}+A_{2} X_{2} \text {, }
$$

where $A_{1}$ and $A_{2}$ are the mean molecular areas of components 1 and 2 , respectively. $X_{1}$ and $X_{2}$ are their mole fraction. If the experimental plot follows the ideal mixing line, it demonstrates a completely miscible or homogenous monolayer where the components mix but do not interact. In addition, it also can imply that the two compositions are immiscible, essentially patches of one composition in a monolayer of the other [29]. The evidence of miscibility with molecular interactions between the components can be deduced from the deviations from the ideal mixing line.

Figure 3 shows the mean molecular area as a function of composition for mixed DPPG/HD monolayers on water subphase at representative surface pressure. The solid lines show that the two components are mixed with effective states. The straight dotted lines present that the two kinds of components are "ideal mixtures" with theoretical area or immiscible. Since a linear relationship between experimental mean molecular area and composition is not satisfied completely, DPPG/HD were considered to be miscible and interact with each other inside the monolayer, the nonideal monolayers formed at the interface. Figure 3 also show that the mean molecular areas decrease with the increment of mole fraction of HD or surface pressures. It should be emphasized that $X_{\mathrm{HD}}$ and surface pressure have an important influence on the interactions between DPPG and HD molecules.
To perform a further analysis of the miscibility of the binary components, $A_{\text {exc }}$ at a given surface pressure was calculated according to (2) $[8,17,25]$. Usually, $A_{\text {exc }}$ can be used for elucidating different molecular interaction and lateral packing as an important physical parameter as well:

$$
A_{\text {exc }}=A-A_{\text {id }} \text {. }
$$

$A$ is the mean molecule area of a mixed monolayer at a given surface pressure obtained experimentally. $A_{\text {id }}$ shows ideal mixed mean molecular area calculated from (1). If $A_{\text {exc }}=0$, it means perfectly miscible or completely immiscible mixed monolayer is observed, and a plot of $A$ as a function of $X_{1}\left(\right.$ or $X_{2}$ ) at a given surface pressure would be a straight line, and further measurements are needed to distinguish these two monolayer states. On the contrary, $A_{\text {exc }} \neq 0$ indicates the miscibility of the mixture and various types of interactions occur in the film $[8,24,25]$. As a matter of fact, there always exist intermolecular forces between the molecules in a monolayer. It is a matter of magnitude of forces that determines $A_{\text {exc }}$ [26]. With regard to binary monolayer, the negative $A_{\text {exc }}$ will be obtained if the attractive intermolecular forces exist. On the other hand, if $A_{\text {exc }}$ is positive, it suggests that the interactions between the two components are repulsive [30].

The $A_{\text {exc }}$ values for DPPG/HD binary mixed system have been presented as a function of mole fraction of HD in Table 1. As shown in Table 1, one can conclude that the condensation effect of HD was sensitive to the monolayer composition and surface pressure. At $\pi=5 \mathrm{mN} / \mathrm{m}$, the deviations are negative for all range of $X_{\mathrm{HD}}$ indicating the molecular interaction is attractive force and the two components are miscible easily. The effect is most prominent when $X_{\mathrm{HD}}=0.4$. For higher surface pressures $(15 \leq \pi \leq 45 \mathrm{mN} / \mathrm{m})$, the condensing effect is predominant at the same concentration of $X_{\mathrm{HD}} \approx 0.8$ (the 
TABLE 1: Excess molecular area as a function of mole fraction of HD at serials discrete surface pressures.

\begin{tabular}{lccccc}
\hline \multirow{2}{*}{$\begin{array}{l}\text { Mole fraction, } \\
X_{\mathrm{HD}}\end{array}$} & \multicolumn{5}{c}{$\pi(\mathrm{mN} / \mathrm{m})$} \\
& 5 & 15 & 25 & 35 & 45 \\
\hline 0.0 & 0 & 0 & 0 & 0 & 0 \\
0.2 & -3.18 & 0.51 & 1.65 & 1.93 & 2.43 \\
0.4 & -5.18 & 0.29 & 1.51 & 1.82 & 2.13 \\
0.6 & -3.27 & 0.33 & 0.58 & 0.05 & -0.91 \\
0.8 & -0.71 & -0.49 & -0.88 & -1.46 & -2.10 \\
1.0 & 0 & 0 & 0 & 0 & 0 \\
\hline
\end{tabular}

point where the minima occur) despite the various surface pressures (see Table 1), but the effect is weaker than that $\pi=5 \mathrm{mN} / \mathrm{m}$ when $X_{\mathrm{HD}}=0.4$. For lower mole fraction of HD, the positive $A_{\text {exc }}$ observed at $15 \leq \pi \leq 45 \mathrm{mN} / \mathrm{m}$ suggests the existence of repulsive fore between DPPG and HD molecules, and the two components may be miscible difficultly.

3.3. Compressibility of Binary Monolayer. To better characterize and elucidate the details of the influence of hexadecanol molecules on the physical state of lipid monolayer, the compression modulus $C_{s}^{-1}$ of the mixed films was studied, defined as (3) [31]:

$$
C_{s}^{-1}=-A\left(\frac{\partial \pi}{\partial A}\right)
$$

$C_{s}^{-1}$ is obtained by numerical calculation of the slope of the $\pi-A$ isotherms $[15,32]$ and plotted as a function of surface pressure (see Figure 4). The modulus is zero corresponding to clean air-water interface and increases with the total mole of surfactant present at the interface. In addition, the value of compression modulus depends on the state of the film, being larger for more condensed monolayers. Higher modulus values related to lower interfacial elasticity $[15,26,31,32]$. A feature minimum on the $C_{s}^{-1}-\pi$ curve was used to identify the LE-LC phase transition [33]. As shown in Figure 4, the minimum $\left(C_{s}^{-1}=16 \mathrm{mN} / \mathrm{m}\right)$ for pure DPPG at $\pi=12 \mathrm{mN} / \mathrm{m}$ indicates the transition from LE to LC, evidenced by the plateau observed in the $\pi$-A isotherm (see Figure 2). During the incorporation of HD molecules into lipid monolayer the minimum moves to lower surface pressure and disappears above $X_{\mathrm{HD}}>0.4$, corroborated by the lack of corresponding features in the $C_{s}^{-1}-\pi$ profiles. According to the criterion given by Davies and Rideal [34] the modulus values distribute from 10 to $50 \mathrm{mN} / \mathrm{m}$ for liquid-expanded phase (LE) and from 100 to $250 \mathrm{mN} / \mathrm{m}$ for liquid-condensed phase (LC) while it distribute above $250 \mathrm{mN} / \mathrm{m}$ for solid phase (S). Figure 4 illustrates that the jumping-off surface pressure of phase transition from LE to LC for DPPG/HD system decreases from 15.2 to $2.5 \mathrm{mN} / \mathrm{m}$ gradually with the mole fraction $X_{\mathrm{HD}}$ increasing from 0 to 1 . Nevertheless, the phase transitions end at surface pressure about $17.5 \mathrm{mN} / \mathrm{m}$ for all mole fractions of HD. It is also worth noting that the $C_{s}^{-1}$ values preponderate over $250 \mathrm{mN} / \mathrm{m}$ at $0 \leq X_{\mathrm{HD}} \leq 0.4$

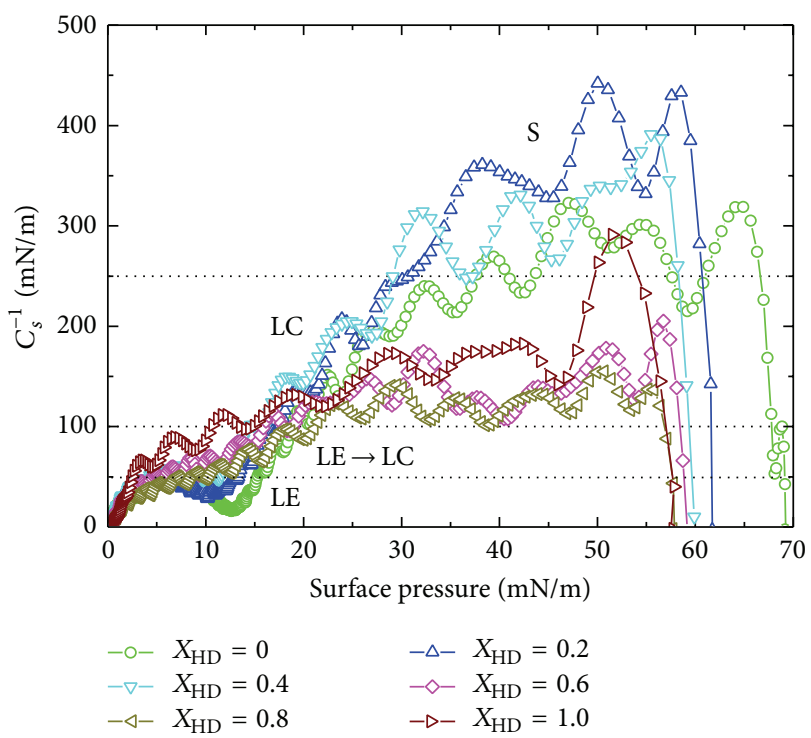

FIGURE 4: The surface compressional modulus as a function of composition for HD/DPPG binary system.

indicating the formation of DPPG/HD solid film, and the compressibility of the binary film is very low. But in the range of $X_{\mathrm{HD}}>0.4$, the LC films observed from the end of LE-LC transition to collapse occurred.

3.4. Thermodynamic Stability Analysis of the Binary Monolayers. The interactions between molecules and the stabilization of monolayer films were evaluated quantitatively by mixed monolayer thermodynamic properties in terms of excess Gibbs free energies function. $\Delta G_{\mathrm{exc}}^{\pi}$ at a series of discrete surface pressure can be estimated quantitatively from the isotherm data points via $(4)[8,30]$ :

$$
\Delta G_{\mathrm{exc}}^{\pi}=N \int_{0}^{\pi}\left(A_{\exp }-X_{1} A_{1}-X_{2} A_{2}\right) \mathrm{d} \pi .
$$

If the monolayer is ideally mixed or totally immiscible, $\Delta G_{\text {exc }}^{\pi}=0$. On the other hand, the positive and negative excess values of the Gibbs free energy indicate the existence of repulsive and attractive interaction force between the two component molecules of the binary system, respectively; that is to say, $\Delta G_{\mathrm{exc}}^{\pi}<0$ indicates the binary monolayer's stability, while $\Delta G_{\mathrm{exc}}^{\pi}>0$ suggests phase separation in the monolayer [25]. In particular, the appearance of a minimum of $\Delta G_{\mathrm{exc}}^{\pi}$ will demonstrate a mixed monolayer of the largest thermodynamic stability in comparison with the pure component monolayers [26]. The Gibbs energy change in the binary mixing system $\left(\Delta G_{\text {mix }}^{\pi}\right)$ can be interpreted as $\Delta G_{\text {mix }}^{\pi}=\Delta G_{\text {id }}^{\pi}-$ $\Delta G_{\text {exc }}^{\pi}$. For ideal mixing, the Gibbs energy change involves only the entropy terms as $\Delta G_{\text {id }}^{\pi}=R T\left(X_{1} \ln X_{1}+X_{2} \ln X_{2}\right)$, where $R$ is the gas constant and $T$ is the absolute temperature.

Figure 5 shows the excess Gibbs energy of mixing as a function of composition for the DPPG/HD mixed monolayer at five considered surface pressures. It is evident that the $\Delta G_{\mathrm{exc}}^{\pi}$ values versus monolayer composition plots show 


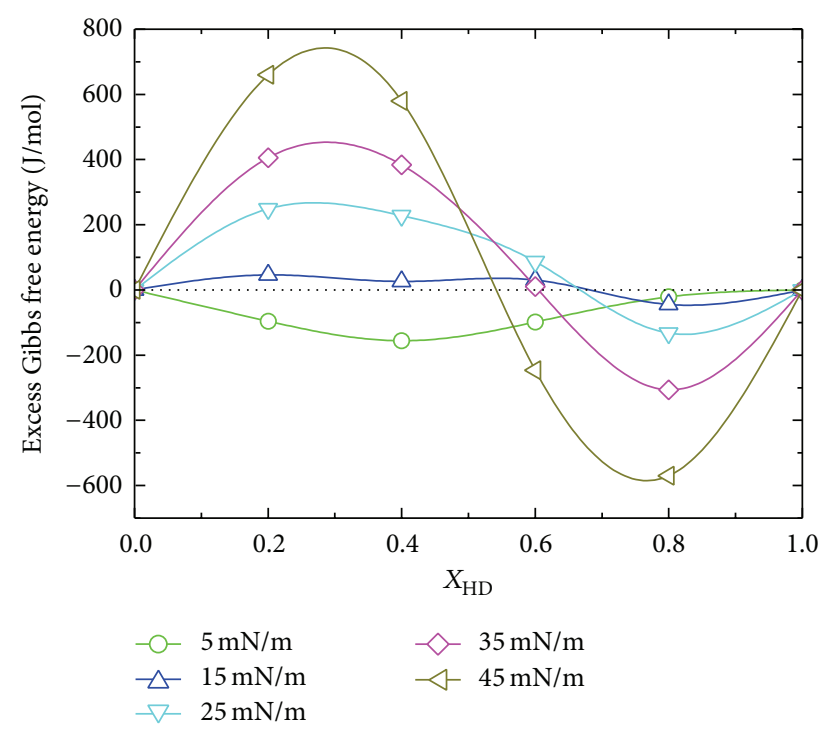

FIgURE 5: The excess Gibbs free energy of DPPG/HD twocomponent systems as a function of the composition of $\mathrm{HD}$ at five definite pressures.

deviation from linearity in the whole range of mole fraction of $\mathrm{HD}$ and proves the existence of the interaction between the molecules. As for DPPG/HD system, negative $\Delta G_{\text {exc }}^{\pi}$ values are obtained at $\pi=5 \mathrm{mN} / \mathrm{m}$ for all $X_{\mathrm{HD}}$, and the minimum (about $-156 \mathrm{~J} / \mathrm{mol}$ ) corresponds to $X_{\mathrm{HD}}=0.4$. The $\Delta G_{\text {exc }}^{\pi}$ values do approach zero over the whole mole fraction range at $\pi=15 \mathrm{mN} / \mathrm{m}$ and this implied that the two compositions mixed may be near ideality. At other surface pressures $(\pi=25-45 \mathrm{mN} / \mathrm{m})$, positive as well as negative $\Delta G_{\text {exc }}^{\pi}$ values were observed, and the maximum and minimum values of $\Delta G_{\text {exc }}^{\pi}$ correspond to $X_{\mathrm{HD}}=0.2-0.3$ and $X_{\mathrm{HD}}=0.7-0.8$, respectively. This suggests that the attractive interaction between monolayer molecules occurs at high HD mole fraction, and the mixed film is more stable.

In general, for DPPG/HD binary system, the trend of $\Delta G_{\mathrm{exc}}^{\pi}-X_{\mathrm{HD}}$ curves are similar to each other at $\pi=15-$ $45 \mathrm{mN} / \mathrm{m}$. With increasing of mole fraction of $\mathrm{HD}$ in the binary system, the positive value of Gibbs free energy occurs, meaning that the lateral phase separation exists and the repulsive interaction between the molecules presents [1, 25]. Negative $\Delta G_{\text {exc }}^{\pi}$ values occur at high concentration of $\mathrm{HD}$, indicating that the interaction between DPPG and HD molecules is attractive, which is favorable for the occurrence of molecular condensation and enhancement of thermodynamic stability of the film system. It is implied that the polar headgroup of DPPG closely integrates with that of HD, which makes the intermolecular attraction stronger.

The interaction energy parameter $\xi$ and activity coefficient $\gamma_{i}(i=1,2)$ of HD and DPPG in the binary monolayer can be evaluated as well from the values of $\Delta G_{\text {exc }}^{\pi}$. Both $\xi$ and $\gamma_{i}$ are two significant parameters for quantitative analysis thermodynamic properties and stability of monolayers. Following the analysis given by Kodama et al. [35], in the framework of the regular solution theory [25], $\xi$ can be obtained using the following equation:

$$
\xi=\frac{\Delta G_{\mathrm{exc}}^{\pi}}{R T\left(X_{1} X_{2}^{2}+X_{2} X_{1}^{2}\right)}=\frac{\Delta G_{\mathrm{exc}}^{\pi}}{R T X_{1} X_{2}} .
$$

The values of activity coefficient $\gamma_{i}$ can be obtained from Margules equations [36] for given binary monolayer systems as follows:

$$
\begin{aligned}
& \ln \gamma_{1}=\xi X_{2}^{2} \\
& \ln \gamma_{2}=\xi X_{1}^{1}
\end{aligned}
$$

The adhesive interactions related to cohesive interaction between dissimilar molecules can be measured by the unitless interaction energy parameter $\xi$ [9]. The negative sign of $\xi$ indicates the molecular interactions of the films become more strongly attractive comparing with single component monolayer while the positive sign of $\xi$ means phase separation and repulsion force between lipid and HD [12].

The calculated values of interaction parameter $\xi$ for the binary systems are listed in Table 2 . The $\xi$ values are positive or negative relying on whether the $\Delta G_{\mathrm{exc}}^{\pi}$ values are positive or negative (see Figure 5). Distinctively, the bigger value of $\xi$ relates to the stronger interaction, and its composition dependence is corresponding to the packing of a considered molecule surrounded by the other molecules [37]. Attention should be paid to the $\xi$ values at $45 \mathrm{mN} / \mathrm{m}$ when $X_{\mathrm{HD}}=0.8$ which is the greatest negative value for all the mixtures, indicating that the HD molecule as the majority can interact most attractively with DPPG molecules as the minority, respectively. This situation is reflected by the activity coefficients as well. Comparing $\gamma_{1}$ and $\gamma_{2}$ values (see Figures 6(a) and 6(b)) at $X_{\mathrm{HD}}=0.8, \gamma_{1}$ is just about one (unity), while the values of $\gamma_{2}$ decrease markedly with the increasing of surface pressure; particularly at $\pi=45 \mathrm{mN} / \mathrm{m}$, the values of $\gamma_{1}$ equal 0.95 while $\gamma_{2}$ fall to 0.4 from about unity at $\pi=5 \mathrm{mN} / \mathrm{m}$. Generally, the interaction parameters increase as the addition of surface pressures at all mole fractions is concerned (see Table 2), which indicates the intermolecular interactions between HD and DPPG strengthen with the improvement of surface pressure.

3.5. Atomic Force Microscopy. AFM observations of LB films transferred onto mica have been performed to characterize aggregation phase behavior and nanostructure of films, which is beneficial for confirmation of the miscibility and molecular interactions of two components for the present systems at the nanoscale level [38]. Earlier reports have reported the physiologically relevant lung surface pressure from 40 to $70 \mathrm{mN} / \mathrm{m}$. [39], so the surface pressure of $45 \mathrm{mN} / \mathrm{m}$ and scanning range of $5 \times 5 \mu \mathrm{m}$ are chosen for all AFM experiments. The AFM morphology images of monolayers of DPPG and HD in six different stoichiometries on the subphase of pure water are shown in Figures 7(a)-7(f). For single component monolayers, as shown in Figure 7 (a) for DPPG and Figure 7(f) for HD, they show a uniform pattern with many small holes and larger platforms, forming defective nanostructures. With the increase of mole fraction 


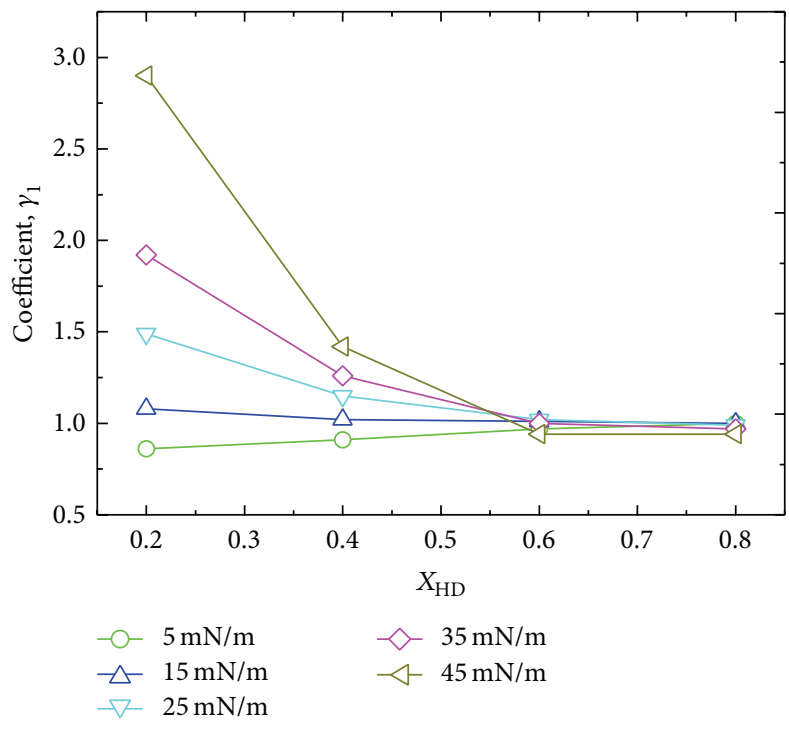

(a)

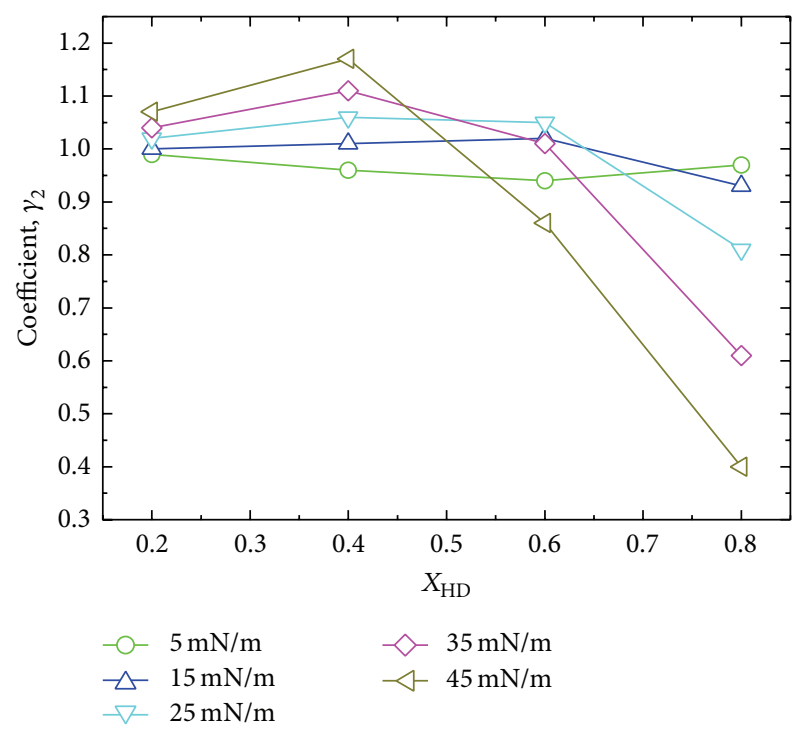

(b)

FIGURE 6: Activity coefficient (a) $\gamma_{1}$ (for HD) and (b) $\gamma_{2}$ (for DPPG) of the mixed monolayers of HD/DPPG.

of $\mathrm{HD}$ in the mixed film, the AFM images at $45 \mathrm{mN} / \mathrm{m}$ (see Figures 7(b) and 7(c)) exhibit two different phases. Judging from the height difference between DPPG and HD, the bight (higher) domain consists of DPPG monolayers (i) and the dark (lower) domain is made of HD monolayers (ii) [40]. This phase separation means that the intermolecular interactions between DPPG and HD are repulsive at lower mole fraction of $\mathrm{HD}$, as is mentioned above (Table 1). On the other hand, the excess Gibbs free energy positive for $X_{\mathrm{HD}}=0.2$ and 0.4 (see Figure 5) implies the monolayers (Figures 7(b) and $7(\mathrm{c})$ ) become less stable. When $X_{\mathrm{HD}}$ is up to $0.6, A_{\mathrm{exc}}=$ $0.05 \mathrm{~nm}^{-2}$; that is to say, the experimental plot almost follows the ideal mixing line and the two components are miscible in the monolayer. This result can be observed in the AFM image (see Figure 7(d)), and from the figure we can see that there is only very small area corresponding to separation phase area. The homologous region (iii) represents that the DPPG and HD are miscible and form LC monolayer (see Figure 4). As shown in Figure 5, the value of $\Delta G_{\text {exc }}^{\pi}$ becomes minimum at the HD mole fraction of 0.8 for surface pressure ranging from 15 to $45 \mathrm{mN} / \mathrm{m}$. This result interprets that the attractive intermolecular interaction between DPPG and HD is strong at this proportion, and as mentioned above, the polar headgroup of DPPG closely integrates with that of $\mathrm{HD}$, so as to cause formation of stable homologous monolayer film (see Figure 7(e)).

\section{Conclusion}

In this paper, the miscibility, stability, intermolecular interaction, and nanostructure of monolayer of DPPG/HD binary mixed system in two-dimensional states were investigated by using the method of LB and AFM technology. For the mixed DPPG/HD systems, this study shows that the two components in the binary system are miscible and formed
TABLE 2: The interaction parameter as a function of mole fraction of $\mathrm{HD}$ at serials discrete surface pressures.

\begin{tabular}{lccccc}
\hline Mole fraction, & \multicolumn{5}{c}{ Interaction parameter } \\
$X_{\mathrm{HD}}$ & 5 & 15 & 25 & 35 & 45 \\
\hline 0.0 & & & & & \\
0.2 & -0.24 & 0.12 & 0.63 & 1.02 & 1.67 \\
0.4 & -0.26 & 0.04 & 0.38 & 0.65 & 0.97 \\
0.6 & -0.17 & 0.05 & 0.15 & 0.02 & -0.41 \\
0.8 & -0.05 & -0.11 & -0.33 & -0.77 & -1.44 \\
1.0 & & & & & \\
\hline
\end{tabular}

nonideal monolayers at the air/water interface from the analysis of surface pressure-area isotherms. The nonideality of these mixed systems is evident in the excess mean molecular area-composition figures, in which the experimental curves of mixed monolayers exhibit somewhat deviation from the ideal ones. According to the results of excess Gibbs energy analysis, the stability of mixed DPPG/HD binary monolayer systems was analyzed and the mixed monolayer with $X_{\mathrm{HD}}=$ 0.8 (but $X_{\mathrm{HD}}=0.4$ at surface pressure equal to $5 \mathrm{mN} / \mathrm{m}$ ) appears to be the most stable. Moreover, the interactions among molecules and aggregation state in the monolayers were analyzed according to the results of compressibility and the excess Gibbs energy. Based on the results of excess Gibbs free energy analysis, the interaction parameter $(\xi)$ and activity coefficients of the respective components $\left(\gamma_{1}\right.$ and $\left.\gamma_{2}\right)$ in the binary monolayers (2D phase) at various mole fractions were calculated. The results distinctly manifest that $A_{\text {exc }}$, $\Delta G_{\text {exc }}^{\pi}$ and $\xi$ of DPPG/HD mixed monolayers get the negative deviations for $X_{\mathrm{HD}}=0.8$ at all range of surface pressure, suggesting an intermolecular attractive interaction exists and 


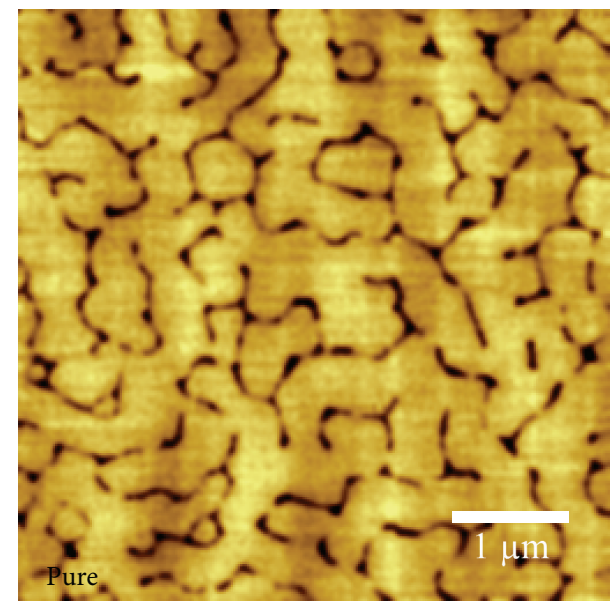

(a)



(c)



(e)



(b)

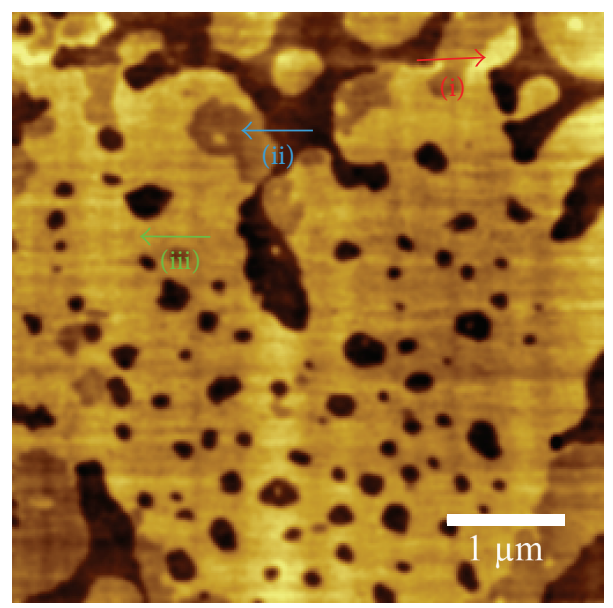

(d)

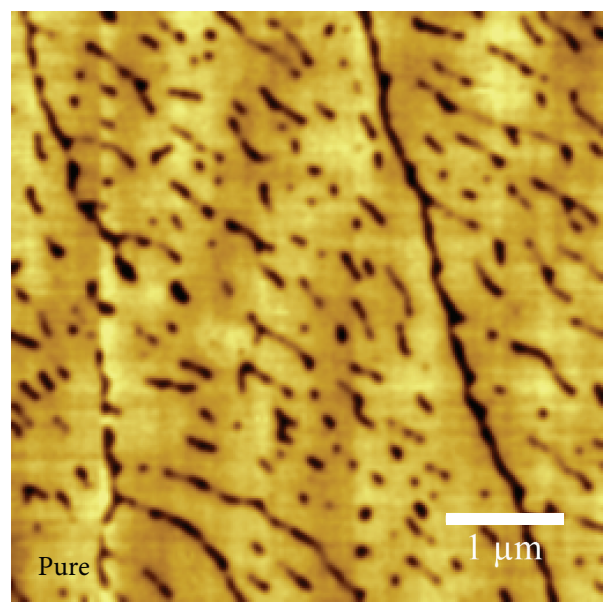

(f)

FIGURE 7: The AFM images of mixed DPPG/HD monolayers for different mole fractions on subphase of pure water: (a) pure DPPG, (b) $X_{\mathrm{HD}}=0.2$, (c) $X_{\mathrm{HD}}=0.4$, (d) $X_{\mathrm{HD}}=0.6$, (e) $X_{\mathrm{HD}}=0.8$, and (f) pure HD, $5 \mu \mathrm{m} \times 5 \mu \mathrm{m}$, transferred on mica at $45 \mathrm{mN} / \mathrm{m}$. 
the binary systems are stable. On the other hand, the positive deviations obtained and increased with the increment of surface pressure from 15 to $45 \mathrm{mN} / \mathrm{m}$ for $X_{\mathrm{HD}}=0.2$ and 0.4 , suggesting an intermolecular repulsive interaction exists and the binary systems are metastable. The interactions between components probably affect and improve the surface tension kinetics of lung surfactants. AFM study for the present paper provided both topography and phase contrast images. The topography images reflect the monolayer topography, while the phase contrast image, which is originated from the energy loss of the oscillating AFM tip, shows the chemical structures of heterogeneous samples [40].

\section{Conflict of Interests}

The authors declare that there is no conflict of interests regarding the publication of this paper.

\section{Acknowledgments}

The work was financially supported by the National Natural Science Foundation of China (no. 21402114, no. 61201088, and no. 11404257), the Shaanxi Province Innovation Project for Science and Technology Overall Planning (no. 2012KTCL0112), the Industrialization Foundation of Shaanxi Educational Committee (no. 2011JG10), and the Industrial Public Relation Project of Shaanxi Technology Committee (no. 2015GY182).

\section{References}

[1] S. Johnson, W. Liu, G. Thakur et al., "Surface chemistry and spectroscopy of human insulin Langmuir monolayer," The Journal of Physical Chemistry B, vol. 116, no. 34, pp. 10205-10212, 2012.

[2] S. N. Ahmed, D. A. Brown, and E. London, "On the origin of sphingolipid/cholesterol-rich detergent-insoluble cell membranes: physiological concentrations of cholesterol and sphingolipid induce formation of a detergent-insoluble, liquidordered lipid phase in model membranes," Biochemistry, vol. 36, no. 36, pp. 10944-10953, 1997.

[3] A. V. Samsonov, I. Mihalyov, and F. S. Cohen, "Characterization of cholesterol-sphingomyelin domains and their dynamics in bilayer membranes," Biophysical Journal, vol. 81, no. 3, pp. 14861500, 2001.

[4] S. L. Veatch and S. L. Keller, "Separation of liquid phases in giant vesicles of ternary mixtures of phospholipids and cholesterol," Biophysical Journal, vol. 85, no. 5, pp. 3074-3083, 2003.

[5] S. L. Veatch, I. V. Polozov, K. Gawrisch, and S. L. Keller, "Liquid domains in vesicles investigated by NMR and fluorescence microscopy," Biophysical Journal, vol. 86, no. 5, pp. 2910-2922, 2004.

[6] K. E. Kirat, S. Morandat, and Y. F. Dufrêne, "Nanoscale analysis of supported lipid bilayers using atomic force microscopy," Biochimica et Biophysica Acta-Biomembranes, vol. 1798, no. 4, pp. 750-765, 2010.

[7] R. E. Kurtz, M. F. Toney, J. A. Pople et al., "Langmuir monolayers of straight-chain and branched hexadecanol and eicosanol mixtures," Langmuir, vol. 24, no. 24, pp. 14005-14014, 2008.

[8] G. He, R. Sun, C. Hao, J. Yang, M. Wang, and L. Zhang, "Thermodynamic analysis and AFM study of the interaction of palmitic acid with DPPE in Langmuir monolayers," Colloids and Surfaces A: Physicochemical and Engineering Aspects, vol. 441, pp. 184-194, 2014.

[9] G. A. Lawrie, I. R. Gentle, and G. T. Barnes, "The structure of mixed monolayer films of DPPC and hexadecanol," Colloids and Surfaces A: Physicochemical and Engineering Aspects, vol. 171, no. 1-3, pp. 217-224, 2000.

[10] C. Alonso, F. Bringezu, G. Brezesinski, A. J. Waring, and J. A. Zasadzinski, "Modifying calf lung surfactant by hexadecanol," Langmuir, vol. 21, no. 3, pp. 1028-1035, 2005.

[11] K.-B. Chen, C.-H. Chang, Y.-M. Yang, and J.-R. Maa, "On the interaction of dipalmitoyl phosphatidylcholine with normal long-chain alcohols in a mixed monolayer: a thermodynamic study," Colloids and Surfaces A: Physicochemical and Engineering Aspects, vol. 170, no. 2-3, pp. 199-208, 2000.

[12] H. Yokoyama, H. Nakahara, and O. Shibata, "Miscibility and phase behavior of DPPG and perfluorocarboxylic acids at the air-water interface," Chemistry and Physics of Lipids, vol. 161, no. 2, pp. 103-114, 2009.

[13] Y. Y. Zuo, R. A. W. Veldhuizen, A. W. Neumann, N. O. Petersen, and F. Possmayer, "Current perspectives in pulmonary surfactant-inhibition, enhancement and evaluation," Biochimica et Biophysica Acta-Biomembranes, vol. 1778, no. 10, pp. 1947-1977, 2008.

[14] G. Wackerbauer, I. Weis, and G. Schwarz, "Preferential partitioning of melittin into the air/water interface: structural and thermodynamic implications," Biophysical Journal, vol. 71, no. 3, pp. 1422-1427, 1996.

[15] A. Gopal and K. Y. C. Lee, "Headgroup percolation and collapse of condensed langmuir monolayers," Journal of Physical Chemistry B, vol. 110, no. 44, pp. 22079-22087, 2006.

[16] T. Jiao, R. Xing, Q. Zhang, Y. Lv, J. Zhou, and F. Gao, "Self-assembly, interfacial nanostructure, and supramolecular chirality of the Langmuir-Blodgett films of some Schiff base derivatives without alkyl chain," Journal of Nanomaterials, vol. 2013, Article ID 297564, 9 pages, 2013.

[17] C. Hao, R. Sun, and J. Zhang, "Mixed monolayers of DOPC and palmitic acid at the liquid-air interface," Colloids and Surfaces B: Biointerfaces, vol. 112, pp. 441-445, 2013.

[18] F. Neville, M. Cahuzac, O. Konovalov et al., "Lipid headgroup discrimination by antimicrobial peptide LL-37: insight into mechanism of action," Biophysical Journal, vol. 90, no. 4, pp. 1275-1287, 2006.

[19] L. Dubreil, V. Vié, S. Beaufils, D. Marion, and A. Renault, "Aggregation of puroindoline in phospholipid monolayers spread at the air-liquid interface," Biophysical Journal, vol. 85, no. 4, pp. 2650-2660, 2003.

[20] R. I. S. Romão, E. Maçôas, J. M. G. Martinho, and A. M. P. S. Gonçalves da Silva, "Interaction of toremifene with dipalmitoylphosphatidyl-glycerol in monolayers at the air-water interface followed by fluorescence microscopy in Langmuir-Blodgett films," Thin Solid Films, vol. 534, pp. 584-590, 2013.

[21] D. Vollhardt, V. B. Fainerman, and S. Siegel, "Thermodynamic and textural characterization of DPPG phospholipid monolayers," The Journal of Physical Chemistry B, vol. 104, no. 17, pp. 4115-4121, 2000.

[22] S. E. Qaqish, S. G. Urquhart, U. Lanke, S. M. K. Brunet, and M. F. Paige, "Phase separation of palmitic acid and perfluorooctadecanoic acid in mixed langmuir-blodgett monolayer films," Langmuir, vol. 25, no. 13, pp. 7401-7409, 2009. 
[23] P. Dynarowicz-Łątka and K. Kita, "Molecular interaction in mixed monolayers at the air/water interface," Advances in Colloid and Interface Science, vol. 79, no. 1, pp. 1-17, 1999.

[24] M. Mottola, R. V. Vico, M. E. Villanueva, and M. L. Fanani, "Alkyl esters of l-ascorbic acid: stability, surface behaviour and interaction with phospholipid monolayers," Journal of Colloid and Interface Science, vol. 457, no. 1, pp. 232-242, 2015.

[25] Y. Chen, R. Sun, and B. Wang, "Monolayer behavior of binary systems of betulinic acid and cardiolipin: thermodynamic analyses of Langmuir monolayers and AFM study of LangmuirBlodgett monolayers," Journal of Colloid and Interface Science, vol. 353, no. 1, pp. 294-300, 2011.

[26] K. Gong, S.-S. Feng, M. L. Go, and P. H. Soew, "Effects of $\mathrm{pH}$ on the stability and compressibility of DPPC/cholesterol monolayers at the air-water interface," Colloids and Surfaces A: Physicochemical and Engineering Aspects, vol. 207, no. 1-3, pp. 113-125, 2002.

[27] K. Hoda, Y. Ikeda, H. Kawasaki, K. Yamada, R. Higuchi, and O. Shibata, "Mode of interaction of ganglioside Langmuir monolayer originated from echinoderms: three binary systems of ganglioside/DPPC, ganglioside/DMPE, and ganglioside/cholesterol," Colloids and Surfaces B: Biointerfaces, vol. 52, no. 1, pp. 57-75, 2006.

[28] D. O. Shah and J. H. Schulman, "Influence of calcium, cholesterol, and unsaturation on lecithin monolayers," Journal of Lipid Research, vol. 8, no. 3, pp. 215-226, 1967.

[29] S. L. Frey, E. Y. Chi, C. Arratia, J. Majewski, K. Kjaer, and K. Y. C. Lee, "Condensing and fluidizing effects of ganglioside GM1 on phospholipid films," Biophysical Journal, vol. 94, no. 8, pp. 3047-3064, 2008.

[30] R. G. Sun, C. C. Hao, J. Zhang, Y. G. Chang, and C. L. Niu, "A monolayer study on phase behavior and morphology of binary mixtures of sulfatides with DPPC and DPPE," Colloids and Surfaces B: Biointerfaces, vol. 73, no. 2, pp. 161-167, 2009.

[31] M. Jurak and J. M. Conde, "Characterization of the binary mixed monolayers of $\alpha$-tocopherol with phospholipids at the air-water interface," Biochimica et Biophysica Acta, vol. 1828, no. 11, pp. 2410-2418, 2013.

[32] S.-S. Feng, K. Gong, and J. Chew, "Molecular interactions between a lipid and an antineoplastic drug paclitaxel (Taxol) within the lipid monolayer at the air/water interface," Langmuir, vol. 18, no. 10, pp. 4061-4070, 2002.

[33] M. Arczewska and M. Gagoś, "Molecular organization of antibiotic amphotericin B in dipalmitoylphosphatidylcholine monolayers induced by $\mathrm{K}^{+}$and $\mathrm{Na}^{+}$ions: the Langmuir technique study," Biochimica et Biophysica Acta-Biomembranes, vol. 1808, no. 11, pp. 2706-2713, 2011.

[34] J. T. Davies and E. K. Rideal, Interfacial Phenomena, Academic Press, New York, NY, USA, 2nd edition, 1963.

[35] M. Kodama, O. Shibata, S. Nakamura, S. Lee, and G. Sugihara, "A monolayer study on three binary mixed systems of dipalmitoyl phosphatidyl choline with cholesterol, cholestanol and stigmasterol," Colloids and Surfaces B: Biointerfaces, vol. 33, no. 3-4, pp. 211-226, 2004.

[36] M. Savva and S. Acheampong, "The interaction energies of cholesterol and 1,2-dioleoyl-sn-glycero-3- phosphoethanolamine in spread mixed monolayers at the air-water interface," Journal of Physical Chemistry B, vol. 113, no. 29, pp. 9811-9820, 2009.

[37] S. Nagadome, N. S. Suzuki, Y. Mine et al., "Monolayers (Langmuir films) behavior of multi-component systems composed of a bile acid with different sterols and with their 1:1 mixtures," Colloids and Surfaces B: Biointerfaces, vol. 58, no. 2, pp. 121-136, 2007.

[38] Z. Leonenko, E. Finot, and D. Cramb, "AFM study of interaction forces in supported planar DPPC bilayers in the presence of general anesthetic halothane," Biochimica et Biophysica ActaBiomembranes, vol. 1758, no. 4, pp. 487-492, 2006.

[39] H. Zhang, Y. E. Wang, Q. Fan, and Y. Y. Zuo, "On the low surface tension of lung surfactant," Langmuir, vol. 27, no. 13, pp. 83518358, 2011

[40] H. Yokoyama, H. Nakahara, T. Nakagawa, S. Shimono, K. Sueishi, and O. Shibata, "Miscibility behavior of twocomponent monolayers at the air-water interface: perfluorocarboxylic acids and DMPE," Journal of Colloid and Interface Science, vol. 337, no. 1, pp. 191-200, 2009. 

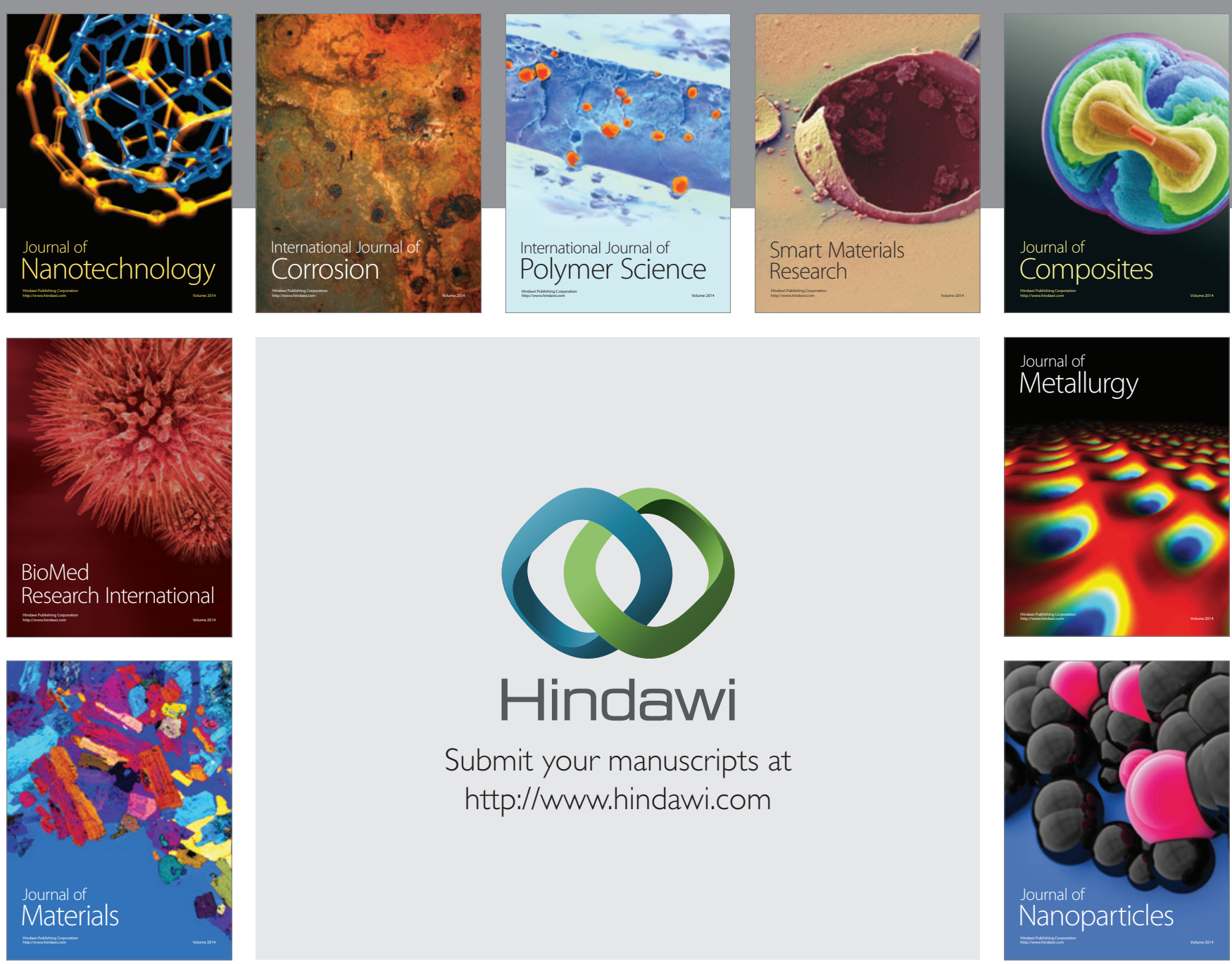

Submit your manuscripts at http://www.hindawi.com
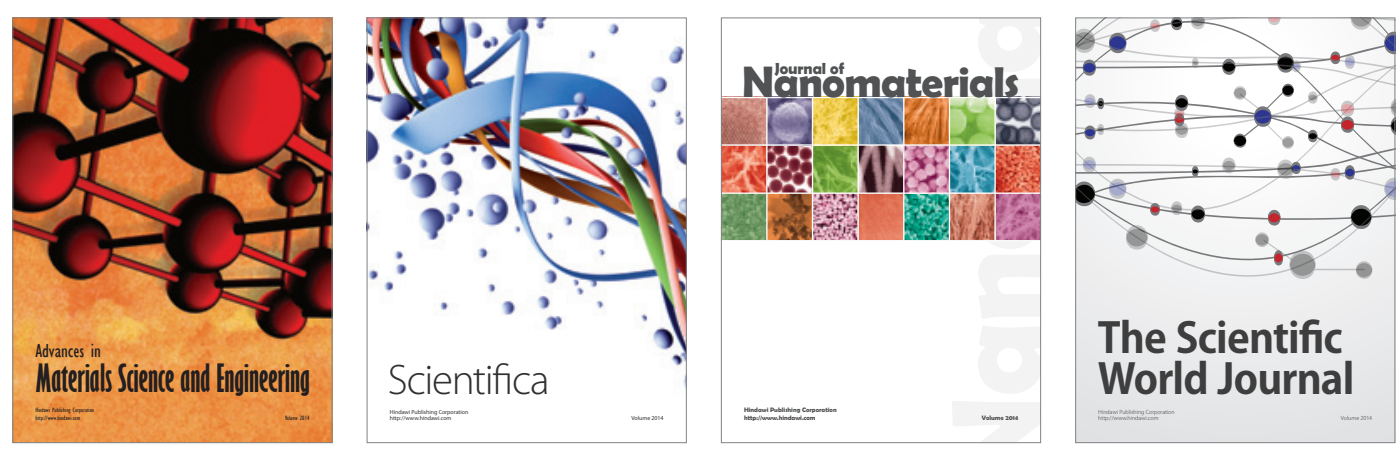

\section{The Scientific World Journal}
